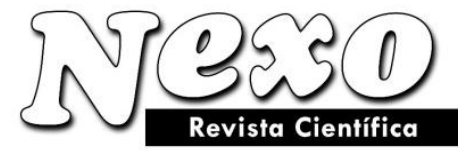

Vol. 25, No. 01, pp.02-08/Junio 2012

\title{
Caracterización de la vegetación arbórea y arbustiva con potencial de servicios ambientales, al este del lago de Nicaragua
}

\author{
E. Salazar*; S. Gámez; N. González; P. Siles \\ ${ }^{1}$ Centro de Investigación y Estudios del Medio Ambiente (CIEMA), \\ Universidad Nacional de Ingeniería (UNI), \\ PO Box 5595, Managua, Nicaragua. \\ E-mail: ciema@uni.edu.ni, stanislaosalazar@yahoo.com.mx
}

(Recibido/received: 21-Marzo-2012; aceptado/accepted: 14-Junio-2012)

\begin{abstract}
RESUMEN
Estudio realizado al Este del Lago de Nicaragua. Para identificar áreas remanentes de bosque, con alto potencial de servicio ambiental. Fueron seleccionados 6 fragmentos de vegetación. Dentro de cada fragmento se identificó la vegetación con menor grado de intervención, y aquí fue establecida una parcela temporal de medición $\left(10,000 \mathrm{~m}^{2}\right)$. Fue levantada información de especie y diámetro medido a la altura del pecho (DAP) para todos los individuos con diámetro mayor a $2.5 \mathrm{~cm}$. Las variables estudiadas fueron: Diversidad, uso y manejo del bosque, carbono almacenado. Los índices de diversidad de Simpson y Shannon-Wiener coinciden en que el fragmento 3 es el más diverso presentando 21 familias, 25 géneros y 30 especies. Fue calculado el número de árboles/hectárea, área basal por hectárea y biomasa por hectárea. Las áreas de bosque proporcionan diversos servicios ambientales. Los índices de biodiversidad sugieren, que la mayoría de estos fragmentos albergan una importante diversidad de especies de árboles y arbustos.
\end{abstract}

Palabras claves: ambiental; bosque; diversidad; Lago; Nicaragua.

\begin{abstract}
This study was carried out east of Lake Nicaragua. To identify remaining areas of forest with high potential for environmental service. Six fragments of vegetation were selected. Within each fragment was identified representative vegetation, and here was established a temporal measurement plot $\left(10,000 \mathrm{~m}^{2}\right)$. Was taken information from species and diameter measured at breast height $(\mathrm{DBH})$ for all individuals with a diameter greater than $2.5 \mathrm{~cm}$. The variables studied were: Diversity, use and management of forest, carbon stocks. Simpson and Shannon-Wiener indices agree that fragment 3 is the most diverse presenting 21 families, 25 genera and 30 species. Forest areas provide various environmental services. Biodiversity indices suggest that most of these fragments harbor an important diversity of species of trees and shrubs.
\end{abstract}

Keywods: diversity, environmental, forest, Lake, Nicaragua

\footnotetext{
* Autor para la correspondencia
} 


\section{INTRODUCCIÓN}

Localizado al Sur Oeste del país, el Lago de Nicaragua es el lago más grande de América Central, con un área de $8,250 \mathrm{Km}^{2}$ está situado a $24 \mathrm{Km}$ del Océano Pacífico y drena en el mar Caribe, en San Juan de Nicaragua, a través del Río San Juan. Es un subsistema del Sistema de la gran Cuenca del Río San Juan. (MADESO (2004). Este subsistema (Lago Cocibolca) es un complejo de ecosistemas acuáticos léntico y lótico y ecosistemas terrestres tales como bosques y humedales. Según MADESO (2004), cerca de 45 ríos drenan al lago de Nicaragua. La mayoría de estos ríos tienen caudal solo en la época lluviosa, sin embargo, durante la estación seca muchos dejan de fluir, es decir no son corrientes permanentes. El área total de drenaje de la cuenca es de $17,300 \mathrm{Km}^{2}$.

Las poblaciones humanas que habitan en esta área ejercen una fuerte presión sobre los recursos naturales, especialmente el suelo, el agua, los bosques y la fauna, y justamente porque es su único medio de subsistencia. Un recorrido rápido de campo en la zona de estudio evidenció una fuerte fragmentación, y pudo visualizarse una gran cantidad de pequeños fragmentos de vegetación casi siempre menores a una hectárea.

El Centro de Investigación y Estudios en Medio Ambiente (CIEMA), identificó y seleccionó la cuenca hidrográfica del Lago de Nicaragua como área de estudio del Proyecto TwinLatin (Hermanando la investigación de cuencas Europeas y Latinoamericanas que hagan posible una gestión sustentable del recurso agua), dentro del marco de aplicación del concepto de la Gestión Integral de Recursos Hídricos (GIRH).

En este contexto, fue reconocida la necesidad de investigar si aún existen áreas importantes de bosque, identificarlas y conocer el estado ecológico en el que se encuentran, para proponer el manejo más adecuado que permita conservar y restaurar estas coberturas forestales indispensables para generar servicios ambientales que tiendan una mejora del Lago de Nicaragua.

Por tanto, el presente trabajo de investigación, precisamente tiene como finalidad proporcionar información técnica y científica sobre las áreas remanentes de bosques, de manera que sirva de base para cualquier proyecto de restauración ecológica tendiente a garantizar una mejora funcional del Subsistema de cuenca Lago de Nicaragua.

\section{METODOLOGÍA}

\section{Ubicación geográfica}

El área del estudio geográficamente está ubicada entre $84^{\circ} 30^{\prime}$ y $85^{\circ} 30^{\prime}$ de Longitud Oeste y $11^{\circ}$ y $12^{\prime}$ de Latitud Norte. Esta área constituye toda la parte Este del subsistema Lago Cocibolca que integra la Cuenca del Río San Juan. Abarca parcialmente a los departamentos de Boaco, Juigalpa, y Río San Juan con altitudes de 70200 msnm para Río San Juan y 100-500 msnm para la zona de Juigalpa y Boaco.

\section{Criterios de selección de las áreas de bosque}

Áreas de bosque natural mayor a 02 hectáreas; formando parte de los sistemas agropecuarios; Con intervención no mayor del $30 \%$ de su cobertura.

\section{Metodología de análisis de los fragmentos}

Dentro de cada fragmento seleccionado se identificó el área con menor perturbación, es decir más conservado, y aquí fue establecida una parcela temporal de medición. La forma de cada parcela de medición fue rectangular con un tamaño de $10,000 \mathrm{~m}^{2}$, cada parcela fue dividida en 10 sub-parcelas de $1000 \mathrm{~m}^{2}$, de las cuales se tomaron tres al azar.

Dentro de estas unidades de medición se registró: El nombre de la especie, diámetro a la altura del pecho (DAP) para todos los individuos con DAP mayor de $2.5 \mathrm{~cm}$. La identificación de las especies arbóreas se realizó en el campo y con la ayuda de guías dendrológicas, de acuerdo a lo recomendado por Acevedo (2005). Las especies que no podían ser identificadas fueron colectadas con material fértil cuando pudo ser posible o con material estéril, para ser identificadas posteriormente en el herbario nacional de la Universidad Centroamericana (UCA-Nicaragua).

\section{Levantamiento de información relativa al manejo de las áreas de bosque}

Fue aplicada una encuesta a los propietarios de las áreas de bosque, con la finalidad de obtener información relevante sobre el uso de las áreas de bosque estudiados, la percepción que los propietarios tienen del área de bosque y de las instituciones nicaragüenses relacionadas a la conservación del Ambiente y los Recursos Naturales. 


\section{Procesamiento y análisis de la información}

Para el procesamiento de información fue utilizado el Software Arcgis versión. 9.2; El Software estadístico para las ciencias sociales (SPSS v. 12). Con el software EstimateS Win versión.6. Se calcularon los índices de diversidad: Chao1, Simpson, Shannon, ICE y ACE.

\section{Análisis de composición florística}

Para comparar la riqueza y diversidad de especies entre los tipos de vegetación fueron utilizados los siguientes estimadores no paramétricos de riqueza de especies tales como Chao1, ACE e ICE (Colwell \& Coddington 1994). Se estimaron los índices de diversidad de Simpson y Shannon para cada una de las parcelas.

La estructura de cada tipo de bosque se resumió en forma descriptiva y en términos de las especies de mayor peso ecológico. Tomando en cuenta la metodología propuesta por Louman et al. (2001) la estructura de la vegetación en las parcelas, fue analizada con base en los valores relativos del área basal, densidad y frecuencia de las especies en las sub-parcelas.

Los valores relativos se combinaron en el índice de valor de importancia (IVI), donde el IVI = (área basal relativa + densidad relativa + frecuencia relativa). En este método el área basal relativa es el área basal de cada especie dividida por el área basal total en la hectárea, la densidad relativa es el número de individuos por especie dividida por el número total de individuos presentes por parcela, la frecuencia relativa es el porcentaje de la frecuencia de parcelas en que está presente la especie con respecto a la suma de frecuencias de todas las especies. Para encontrar patrones estructurales comunes para los tipos de vegetación, fue analizada la estructura poblacional de las especies con IVI más elevado.

Con la información levantada en el muestreo de la vegetación, fue realizada una inferencia estadística por especie para determinar los parámetros estructurales de esa área de bosque (Número de árboles/hectárea, área basal por hectárea, biomasa por hectárea).

Las encuestas realizadas fueron procesadas creando gráficos para indicar la opinión de los dueños de bosques encuestados. Se utilizó el programa Arcgis, para determinar la posición exacta de las áreas de bosque. Fueron calculadas las áreas exactas para cada bosque, y fue elaborado un mapa con estas ubicaciones.
Determinación biomasa total y cantidad de carbono en la biomasa aérea

Se procedió a aplicar la ecuación alométrica desarrollado por el Proyecto LUCCAM (2009), para la zona de San Carlos, Rio San Juan. Esta ecuación es de fácil utilización porque solo requiere la medición de una variable directamente del campo, la cual es el diámetro a la altura del pecho (DAP). Este modelo puede aplicarse a arbustos y árboles de especies forestales con diámetros de 2.5 hasta $100 \mathrm{~cm}$, y toma como principal dato al diámetro medido a $1.30 \mathrm{~m}$ sobre el nivel del terreno, o diámetro a la altura del pecho (DAP) como también suele llamársele. La ecuación estima Kilogramos de materia seca procedente de la biomasa aérea de árboles y arbustos. El modelo es el siguiente:

$$
\ln (\mathrm{Y})=-1.47+\ln 2.72 \ln (\mathrm{X})
$$

Y: Es la biomasa en kilogramos de materia seca $\mathrm{X}$ : Es el DAP en centímetros

Para este proceso fue creada una base de datos en el software Excel, en el cual se insertaron las fórmulas para el cálculo de la biomasa aérea de árboles y arbustos forestales y el carbono almacenado en ellos. El R2 ajustado de esta ecuación de 0.89 .

Con el DAP (Diámetro a la altura del pecho), obtenido de las mediciones en campo, fue creada una base de datos en el Software Excel e introducida la ecuación para los cálculos de la biomasa aérea con la cual se determinó la fracción de carbono en la biomasa aérea y posteriormente fue multiplicada por el factor 0.5 , considerando que según Andrade \& Ibrahim (2003) el carbono es aproximadamente el $50 \%$ del peso seco de un árbol.

\section{RESULTADOS Y DISCUSIÓN}

\section{Fragmentos de bosque}

Fueron identificados seis fragmentos representativos de bosques, de los cuales cuatro están localizadas en el departamento de Río San Juan, uno en Acoyapa Chontales y uno en el Municipio de San Lorenzo, Boaco.

\section{Estado ecológico de las áreas de bosque estudiadas}

Todos los fragmentos de bosque estudiados son bosques secundarios, en su mayoría constituidos por 
especies pioneras, tales como Guazuma ulmifolia, Cecropia peltata, Apeiba tiborbou. Los fragmentos de bosque, están ubicados cerca de comunidades humanas, dentro de fincas dedicadas a la agricultura y a la ganadería. Estas actividades ejercen presión sobre las áreas de bosque, por lo que estos presentan diferentes grados y tipos de perturbación, tales como caminos por donde circulan personas, acceso de ganado a las áreas de bosque, incidencia de incendios forestales, durante la época seca.

\section{Número de especies estimadas}

En base a las especies observadas en cada fragmento de bosque, y en base a los principios en los que se basan los estimadores ACE, ICE, y Chao1, el índice ACE, predice el mayor número de especies para el fragmento 4 , prediciendo 54 especies (tabla 1), mientras que ICE, coincide en que el fragmento 4 es el más diverso, estimando 50 especies. Por su parte Chao 1 confirma que el fragmento 4 es el más diverso, estimando para este, 55 especies forestales (tabla 1 ).

Los estimadores ICE, y Chao1 coinciden en que el fragmento 3 ocupa el segundo lugar en cuanto a riqueza de especies, estimando 40 y 45 especies respectivamente. Estos tres estimadores coinciden en que el fragmento 1 es el menos diverso, coincidiendo en que su riqueza de especies de árboles y arbustos no va más allá de 25 especies diferentes.

Estos resultados, sugieren que los fragmentos de bosque $3,4,5$ y 6 todavía conservan una importante diversidad de especies forestales, sin embargo esta diversidad se encuentra cercana al número mínimo de especies que teóricamente podría esperase en un bosque tropical.

CATIE (2001) expresa que en los bosques tropicales rara vez se encuentra menos de 40 especies forestales por hectárea. En el presente estudio, este es el caso del fragmento de bosque 1, el cual únicamente presenta 25 diferentes especies forestales.

Tabla1. Especies observadas (EO), especies estimadas por ACE, ICE y Chao, para seis fragmentos de bosque, al Este del Lago de Nicaragua

\begin{tabular}{ccccccc} 
Índice/Bosque & B 1 & B2 & B3 & B4 & B5 & B6 \\
& & & & & & \\
\hline EO & 19 & 18 & 28 & 24 & 20 & 22 \\
AC & 25 & 45 & $\mathbf{4 0}$ & $\mathbf{5 4}$ & 35 & 44 \\
ICE & 26 & 41 & $\mathbf{4 5}$ & $\mathbf{5 0}$ & 38 & 40 \\
Chao 1 & 24 & 36 & 40 & $\mathbf{5 5}$ & 28 & 35 \\
\hline
\end{tabular}

Índices de diversidad Shannon-Wienner y Simpson para los fragmento de bosque estudiados

Los índices Shannon-Wienner y Simpson coinciden en que los fragmentos de boque 3, 5 y 6 presentan la mayor abundancia de especies, mientras que encuentran a los fragmentos 1, 2 y 4 como los fragmentos de bosque con la menor abundancia de especies de árboles y arbustos forestales (Tabla 2).

Tabla 2. Índices de diversidad Shannon-Wienner y Simpson para los fragmento de bosque al este del Lago de Nicaragua.

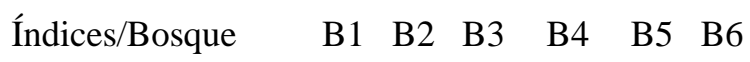

\begin{tabular}{lrrrrrr} 
Simpson & 8 & 7 & 11 & 5 & 11 & 11 \\
Shannon-Wienner & 2.4 & 2.3 & 2.6 & 2.4 & 2.6 & 2.5 \\
\hline
\end{tabular}

Índices de diversidad beta

En este estudio, el índice de Jaccard encuentra que los fragmentos de bosque 1 y 2 , son los más similares, compartiendo 9 especies. Las especies compartidas por estos dos fragmentos de bosque son: Albizia saman (Jac.) Muell, Apeiba tiborbou Aubl. Cecropia peltata L, Cordia alliodora (R.\&P.) Oken., Guazuma ulmifolia, Inga Spp, Lonchocarpus spp1, Lonchocarpus spp2, Spondias mombis L.

\section{Análisis de la estructura}

El fragmento de bosque 3 presenta la mayor densidad, estimando que existen 550 individuos/ha, en segundo lugar el fragmento de bosque 4 , con una densidad de 450 individuos por hectárea. El menor número de individuos, lo presenta el fragmento de bosque 5 estimándose para este 200 individuos por hectárea (tabla3)

Los resultados del presente estudio, en términos de densidad, son muy congruentes con un estudio realizado por Moraes (2001) en San Carlos, Rio San Juan, quien encontró que la densidad promedio de árboles por bosque, varió de 152 a 870 individuos/ha, en bosques de edades similares al de la presente investigación.

\section{Área basal por hectárea}

En cuanto al área basal, el mayor valor lo presenta el fragmento de bosque 1 con $14 \mathrm{~m} 2 / \mathrm{ha}$, seguido del 
fragmento 3 con $13 \mathrm{~m} 2$.ha-1. El menor valor de área basal lo presenta el fragmento de bosque 6 , con $3 \mathrm{~m} 2 / \mathrm{ha}$.

Estos resultados son congruentes con Moraes (2001) quien en un estudio en San Carlos Río San Juan, para el área basal encontró que había diferencias significativas entre bosques, en función de la edad de estos, variando desde $1.9 \mathrm{~m} 2$.ha- 1 en bosques de 6 años de edad hasta $37.7 \mathrm{~m} 2$.ha- 1 en un bosque de 23 años de edad.

Los resultados del presente estudio respecto al área basal, son ligeramente inferiores a los reportados por Gillespie et al (2000), en su estudio sobre la diversidad, composición y estructura del bosque seco tropical en Centro América, los cuales estudiaron siete áreas de bosque bajo conservación y encontraron valores desde $17.7 \mathrm{~m} 2$.ha-1 en la isla de Ometepe, hasta $21.2 \mathrm{~m} 2$.ha-1 en Masaya y Chococente. Es razonable atribuir esta diferencia al hecho de que las áreas estudiadas por Gillespie et al (2000) son áreas bajo conservación.

\section{Potencial de almacenamiento de carbono}

En cuanto a la biomasa, los fragmentos de bosque 1 y 3 son similares, presentando el mayor valor con 60 Mg.ha-1. El menor valor de biomasa lo presenta el fragmento de bosque 6, con $4 \mathrm{Mg}$.ha-1 (fig.4).

En cuanto al carbono almacenado en la biomasa hasta el momento de este estudio, los fragmentos de bosque $1 \mathrm{y}$ 3 , presentan el mayor potencial, y han almacenado 30 $\mathrm{Mg}$ de $\mathrm{C} / \mathrm{Ha}$. El segundo lugar en cuanto a carbono almacenado lo presenta el fragmento de bosque 4, con $24 \mathrm{Mg}$ de C.ha-1 (fig.4).

Tabla 3.Densidad, área basa, biomasa aérea y carbono almacenado para cada fragmento de bosque al Este del Lago de Nicaragua

\begin{tabular}{lcccccc}
\hline Índice/Bosque & B1 & B2 & B3 & B 4 & B5 & B6 \\
\hline Indiv/Ha & 340 & 340 & 550 & 450 & 200 & 300 \\
$\mathrm{Ab}\left(\mathrm{m}^{2}\right)$ & 14 & 7 & 14 & 11 & 4 & 2.5 \\
$\mathrm{BA}\left(\mathrm{Mgha}^{-1}\right)$ & 60 & 30 & 60 & 42 & 19 & 8 \\
$\mathrm{CA}\left({\mathrm{Mg} \mathrm{C} . \mathrm{Ha}^{-1)}}^{-30}\right.$ & 16 & 30 & 22 & 8 & 4 \\
\hline
\end{tabular}

Indiv/Ha: Individuos por hectárea

$\mathrm{Ab}\left(\mathrm{m}^{2}\right)$ : Área basal por hectárea en metros cuadrados

$\mathrm{BA}\left(\mathrm{Mgha}^{-1}\right)$ : Biomasa aérea por hectárea (en mega gramos)

$\mathrm{CA}\left(\mathrm{Mg} \mathrm{C}_{\mathrm{Ha}}{ }^{-1)}\right.$ : Carbono almacenado/hectárea (en mega gramos)

\section{Composición florística}

De un total de 657 taxas para las que se obtuvo información directamente en campo, fueron encontradas 131 especies pertenecientes a 115 géneros agrupados en 103 familias. El fragmento de bosque 3 resultó con el mayor número de especies, géneros y familias, seguido del fragmento de bosque 4, mientras que el fragmento de bosque 2 resultó tener el menor número de especies, géneros y familias.

Las familias y géneros encontrados en estas seis áreas de bosque, son típicas de bosques segundarios, coincidiendo con el estudio de Moraes (2001) para la misma zona de estudio. El presente estudio también coincide con este mismo autor en que muchas de las familias encontradas presentan especies que tienen valor comercial, entre ellas Cordia alliodora, Lonchocarpus sp, Tabebuia roseae, Albizia adinocephala, Albizia saman, Hymenea courbaril, Platymicium sp.

Valor de importancia ecológica de las especies en cada fragmento

En el bosque 1, las especies con mayor índice de valor de importancia fueron Spondias mombis, de la familia anacardiaceae con un IVI de 184, Callycophylum canidisimum de la familia Rubiaceae, con un IVI de 134, Zanthoxilum setulosum perteneciente a la familia Rutaceae, con un IVI de 121 y Codia alliodora, de la familia Boraginaceae con un IVI de 104.

Para el fragmento de bosque 2 Guazuma ulmifolia presenta el mayor índice de valor de importancia siendo de 150, Spondias mombis presenta un IVI de 136, seguido por Cordia alliodora cuyo IVI es de 121, y por Cochlospermum vitifolium que presenta un IVI de 120.

Las especies del fragmento de bosque 3 presentan los siguientes índices de valor de importancia: El mayor valor lo presenta Cecropia peltata con un IVI de 133, seguido de Enterolobium ciclocarpum con un IVI de 133, Acacia collinsii tiene un IVI de 112, y Guazuma ulmifolia también presenta un IVI de 112.

En el fragmento de bosque 4 presenta las especies con mayor IVI son Bravaisia integerrima con 162, Trichilia martiana con 135, Spondias mombis con 123 y Acacia collinsii con un IVI de 119.

En el fragmento de bosque 5 únicamente dos especies presentan un IVI ampliamente superior al resto de las 
especies, Guazuma ulmifolia con un IVI de 135 y Trichilia cuachijuga con un IVI de 94.

En el fragmento de bosque 6, la especie con mayor IVI es Diospyros salicifolia con 134, seguido de Myrospermun frutecens, que presenta un IVI de 133, Gliricidia cepium cuyo IVI es de 133, y Thouinidium decondrum que presenta un IVI de 110.

\section{Uso y manejo de los fragmentos de bosque}

De forma general, el mayor uso dado al suelo de las fincas donde se encuentran los fragmentos de bosque, son las pasturas utilizadas para la ganadería, la cual se realiza de forma extensiva. En menor proporción el suelo es utilizado para agricultura, principalmente granos, raíces y tubérculos. Solo un mínimo porcentaje del suelo de las fincas es destinado para el bosque, el cual proporciona leña y madera para la finca principalmente.

En cuanto a la tenencia de la tierra, las seis fincas donde se ubican los fragmentos de bosque, se encuentran bajo el régimen de propiedad privada individual, con sus respectivos títulos de propiedad.

\section{CONCLUSIONES}

El mayor de los fragmentos de bosque encontrados fue de 32.8 hectáreas y el menor, fue de 2.2 hectáreas. La mayor diversidad de especies de árboles y arbustos de forma general está concentrada en las áreas mayores a 2 hectáreas.

Para el total del área estudiada, el estimador ICE, predice que existen 125 especies, en total en los fragmentos de bosque estudiados. El estimador ACE, predice que existen hasta 125 especies diferentes. El estimador Chao 1, predice que pueden encontrarse hasta 250 diferentes especies de árboles y arbustos. De manera general estos resultados sugieren que estos fragmentos de bosque todavía conservan una diversidad de especies que justifica implementar un programa de manejo y conservación para estos fragmentos de bosque.

De forma general, los índices Shannon-Wiener y Simpson sugieren que los fragmentos de bosque 1 y 5 presentan la menor diversidad de especies arbóreas y arbustivas, en tanto que los fragmentos de bosque 2, 3 , 4 y 6, presentan la mayor diversidad de árboles y arbustos, por lo tanto el programa de manejo y conservación deberá priorizar estos fragmentos de bosque.
En este estudio, los índices de Jaccad y Sorensen, encuentran que los fragmentos de bosque 1 y 2 , son los más similares, compartiendo 9 especies en común. El análisis de cluster mostró dos grupos principales. El primer grupo está conformado por los fragmentos de bosque 1, 2, 3,4 y 5. El segundo únicamente por el fragmento 6. Esto es de especial importancia para el diseño de un programa de conservación de acuerdo a los grupos con mayor similitud.

En cuanto al área basal, el mayor valor lo presenta el fragmento de bosque 1 con $14 \mathrm{~m} 2 / \mathrm{ha}$, seguido del fragmento 3 con $13 \mathrm{~m} 2$.ha-1. El menor valor de área basal lo presenta el fragmento de bosque 6 , con $3 \mathrm{~m} 2 / \mathrm{ha}$. Son valores que pueden esperarse en los bosques secundarios. El programa de manejo y conservación deberá estar orientado a aumentar el área basal por hectárea.

En cuanto al carbono capturado, los fragmentos de bosque 1 y 3 , presentan el mayor almacenamiento, siendo este de $30 \mathrm{Mg}$ de $\mathrm{C} / \mathrm{Ha}$. El segundo lugar en cuanto a captura de carbono lo ocupa el fragmento de bosque 4, con $24 \mathrm{Mg}$ de $\mathrm{C} / \mathrm{Ha}$.

En general estas son áreas de mayor edad (20 años), por lo tanto presentan árboles con diámetros mayores con respecto a los fragmentos con menor carbono almacenado. Un programa que integre el servicio ambiental de almacenamiento de carbono, deberá priorizar estos fragmentos de bosque.

Dentro de las fincas en la que se encuentran inmersos los fragmentos de bosque estudiados, los principales usos que los productores le dan al área donde no existe bosque es el cultivo de granos básicos, y la ganadería. Mientras que el principal uso que le dan al bosque es la extracción de leña y madera para la finca, y como una potencial área agrícola o ganadera. De forma general no se le da ningún tipo de manejo silvicultural al bosque.

Únicamente los propietarios de los parches 2, 3, 4 y 5 actualmente tienen la visión de conservación. En cuanto al uso futuro, únicamente los propietarios de los parches de bosque 2, 3 y 6 mantienen su visión de conservación, por lo que estos propietarios deben considerarse al realizar un proyecto en la zona.

El manejo y conservación de estos remanentes de vegetación es imprescindible para garantizar generación de servicios ambientales, tales como la captura de carbono, la retención de sedimentos, y la conservación de fuentes de agua.

Vol. 25, No. 01, pp. 02-08/Junio 2012 


\section{REFERENCIAS}

Acevedo, R.P.2005. Vines and Climbing plants of Puerto Rico and the Virgin Islands. Contributions from the United States National Herbarium. 51:1-483.

Andrade, H; Ibrahim, M. 2003. Como monitorear el secuestro de carbono em sistemas silvopastoriles. Revista Agroforesterías en las Américas Vol.10 NO 3940.CATIE.Turrialba, CR.127P.

CATIE. 2001. Silvicultura de bosques latifoliados húmedos con énfasis en América Central. Turrialba, CR.263 P.

Colwell, R; Coddington, J. 1994. Estimating terrestrial biodiversity through extrapolation. Phil. Trans. Royal Soc. London B. 345:101-118.

Clench, H.K. 1978. To make regional lists of butterflies some thoughts. Lepidopterists'Society, 33(4): 216-231

Gillespie, T; Grijalva, A; Farris, C. 2000. Diversity, composition, and structure of tropical dry forests in Central America. Plant Ecology. 147:37-47.

Louman, B; Valerio, J; Jimenez, W. 2001. Bases ecologicas. In Silvicultura de bosques latifoliados humedos con enfasis en America Central. Manual tecnico/ CATIE; no 46 Eds. B. Louman, D. Quiros and M. Nilsson. CATIE, Turrialba, CR, pp. 21-75.

MADESO. 2004. Estudio diagnostico de la Cuencas del Rio San Juan.Disponible en:

www.oas.org/dsd/publications/

Moraes, F.Ch.2001. Almacenamiento de carbono en bosque secundarios en el municipio de San Carlos, Nicaragua. CATIE. Turrialba, Costa Rica.100P.

Proyecto LUCCAM. 2009. Allometric models for biomass estimation in secondary forests, San Carlos Municipal District, Nicaragua. Proyecto Cambio de uso de la tierra y flujos de carbono para Centroamerica. CATIE-Universidad de Helsinki, Finlandia. 120 P

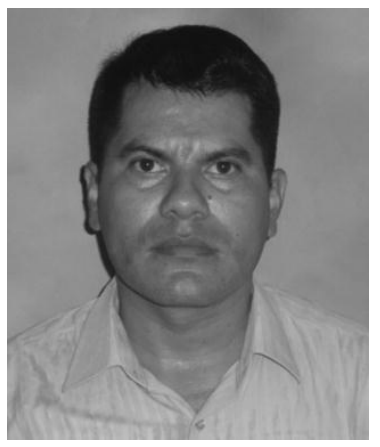

Estanislao Salazar Quiroz, graduado de Ingeniero forestal por la Universidad Nacional Agraria (UNA, Managua) en el año 2003. Graduado en la Maestría en Ciencias Ambientales por el Centro de Investigación y Estudios en Medio Ambiente (CIEMA) de la Universidad Nacional de Ingeniería (UNI). Su campo de investigación es la conservación de la biodiversidad forestal. 